\title{
Food Talks: visual and interaction principles for representing environmental and nutritional food information in augmented reality
}

\author{
Nicolas Henchoz \\ $E P F L+E C A L L a b$
}

\author{
Emily Groves \\ $E P F L+E C A L ~ L a b$
}

\author{
Andreas Sonderegger \\ $E P F L+E C A L L a b$
}

\author{
Delphine Ribes \\ $E P F L+E C A L L a b$
}

\begin{abstract}
This user-centered design research project aimed to investigate visual and interaction principles for augmented reality (AR) in the context of environmental and nutritional food labelling. Nutritional information on existing food labels is often misunderstood and environmental information is seldom depicted, despite consumer demand. The project explored the potential of $\mathrm{AR}$ in this context with a two-phase process. Phase 1 aimed to engage large audiences in public spaces using a stand-alone AR device showing environmental information only. This allowed design strategies to be tested. Phase 2 addressed personalised information, combining nutritional and environmental data with smartphone AR. Here we integrated design learnings from Phase 1 and then focused on assessing the benefits of AR. A betweensubjects study with 84 participants compared two-versions of the smartphone application; one version showed the information with $\mathrm{AR}$ and the other showed the same information with a static page. Results showed that participants using the AR version learned more about food products than those using the static version. In addition, the AR version matched the high scores of the static version with regards to usability (SUS score of 86) and aesthetics (VisAWI score of 5.9), despite technical limitations of AR. This work reveals that AR can be a credible medium in the food industry and provides visual and interaction design learnings to inform designers in the industry.
\end{abstract}

Keywords: Augmented reality. Food labelling. User experience. Interaction design. Design research.

Index Terms: Human-centered computing Mixed / augmented reality - Human-centered computing User studies • Humancentered computing User centered design • Human-centered computing Empirical studies in interaction design

\section{INTRODUCTION}

With a rise in food allergies [24] and changing eating habits [21] [31], diets are becoming more diverse and specific. In addition, customers are becoming increasingly aware that the production of food requires substantial inputs of land, water, fertilizer, pesticides, and energy [17]. Although food labels provide the main dialogue between a product and consumer [3], they are increasingly considered to be inadequate, with studies showing that the information on food packaging is often difficult to understand [3] or not even read at all [13].

Design research in the field of Augmented Reality (AR) can create new ways to engage people with the immaterial value of physical objects [15]. This led to the hypothesis that AR could be used to address the shortcomings of food labelling. By creating a new user experience, it could directly link data to a food product and thus enhance information perception. Therefore, this research aimed to understand whether presenting food information in AR improved user learning and understanding in comparison with a non-AR, static representation.

\section{Current State of Research}

\subsection{Information Representation in AR}

In 2019, Müller [20] systematically reviewed existing literature and published a conceptual framework for modelling information representation in AR. In this context, two concepts from the framework are relevant; legibility of text and coherence.

Regarding legibility, Palm showed that sans serif fonts stand out against the background more than serif [22], but a study by Gabbard et al. suggests using a "billboard" style, where a background is put behind text for maximum readability [11].

Coherence refers to how well digital AR content is integrated into the real-world environment. This includes aspects such as ensuring that three-dimensional objects are placed at appropriate depths $[10,18]$ or that timing and positioning are optimised $[7,16]$. In addition, Wagner et al. found that adding sound to AR visuals [29] increases user perception of augmented reality. Sugano et al. discuss the benefits of adding shadows to 3D objects to integrate it into a space [27]. Other papers have discussed how nonphotorealistic, or stylised AR content can improve immersion and perception $[8,14,30]$, as the differences between real world and augmented content appear reduced. Finally, observations made by in the field of design research [15] point towards using a "Super Normal" [9] approach for AR which can reduce questioning by users as technological elements (both hardware and software) are integrated into their existing behaviours.

\subsection{AR Usability Studies}

In 2018, Dey et al. [5] systematically reviewed 369 individual AR usability studies published between 2005 to 2014. They reported that the majority of studies are conducted in laboratories, with an average (median) number of participants ranging between 12 and 18 , and with the majority being within-subjects designs. They concluded that more studies with greater numbers of participants and diversity in appraoch was needed.

\subsection{AR in the Food Sector}

We observed that a large number of projects using AR in the food industry were released between 2012 and 2015, where packaging was augmented with marketing information (such as those featured in [32]), intended for short term impact, or a "woweffect". Other projects stemming from the academy test different applications. Rollo et al. [23] created and tested a mobile application prototype called ServAR which aimed to guide the serving size of food. The results showed improved accuracy and consistency of estimating standard serve sizes when using ServAR compared to the information only and control conditions. Tanno et al. [28] developed AR DeepCalorieCam to estimate calorie content of meals through artificial intelligence. This is available as an iOS application but user testing results have not been published. To the best of our knowledge, very few examples of food related AR solutions that are intended for long-term informational use have been published so far. 


\subsection{Information on food packaging}

Solutions to improve nutrition information on food packaging are appearing with the introduction of more colour-coded and simplified systems known as "front of pack" labelling. Comparing to a situation where they are not used, a study showed that the addition of "front of pack" labelling is significantly associated to an increase in consumers' ability to classify products correctly [6]. However, despite consumer interest to have information about the environmental impact of a product on food labels [4,12], it is seldom implemented [17] and if it is, it tends to be both confusing [3] or oversimplified. Indeed, a study by Leach et al. in 2016 [17] into the design of environmental labels was inconclusive, stating a difficulty in finding a balance between options that are simple and easy to understand versus others which give more context but are more difficult to access.

\subsection{Digital food information}

Digital food labels are becoming more common through the introduction of smartphone applications such as Yuka [33], CodeCheck [34] and Open Food Facts [35]. These applications require the user to scan a product's barcode with their smartphone and then a page will appear with information about the product. Although becoming increasingly popular [1], they are mainly limited to nutritional information and customer comments.

\section{Methodology}

\subsection{User-Centred Design Process}

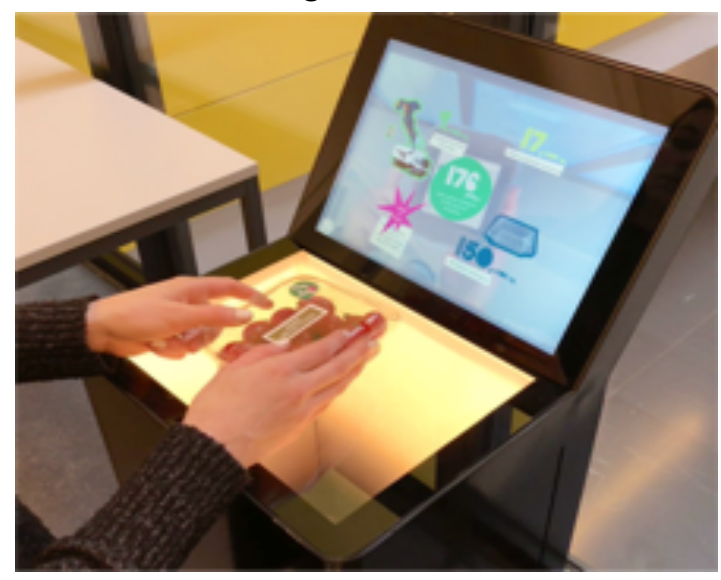

Figure 1: Using the AR Kiosk

The project ran in two phases representing two different levels of engagement. In Phase 1 we designed a prototype for the "Kiosk", a standalone AR device, showing only environmental information. The Kiosk allows AR technology to be brought into the context of the supermarket without customers having to use their own mobile phone. The interactions (see Figure 1) are also based on the notion of Super Normality, using familiar gestures and practices used in the supermarket environment for self-checkouts or weighing scales. We only showed environmental information based on the hypothesis that nutritional and personalised information could be embarrassing for people in a public space.

We ran a workshop with 5 designers to generate initial visual and interaction principles for the Kiosk. We then showed them to 30 customers in the real supermarket environment and asked for their feedback. The majority of them preferred a direct overview of information over an animation or interaction. Modular and icon-based visual styles were also ranked higher than more traditional grid or graph layouts. We used this feedback to develop a final solution which was again qualitatively tested in the supermarket environment.

In Phase 2, visual and interaction principles from Phase 1 were transferred to the mobile context. Two layers of information were defined. The first was an overview combining environmental, nutritional, personal and provenance information which appears as soon as an object is recognised. The second layer provides more detail on each of those four modules when tapped. The scenario of use also changed from the supermarket to the home based on several hypotheses. Firstly, that mobile interactions would require a greater time investment which customers do not have in the supermarket. Secondly, that customers mainly make quick decisions and repeat purchases in the supermarket, and have more time for reflection at home. And finally, because nutritional information is more personal and less suited to public space.

The visual design integrates several techniques from the literature such as the addition of sound for coherence [29], the "billboard" style for text legibility [11] and a non-photorealistic style $[8,14,30]$, using the metaphor of a paper collage. It also uses icons and non-numeric indicators in a similar way to existing front-of-pack labelling.
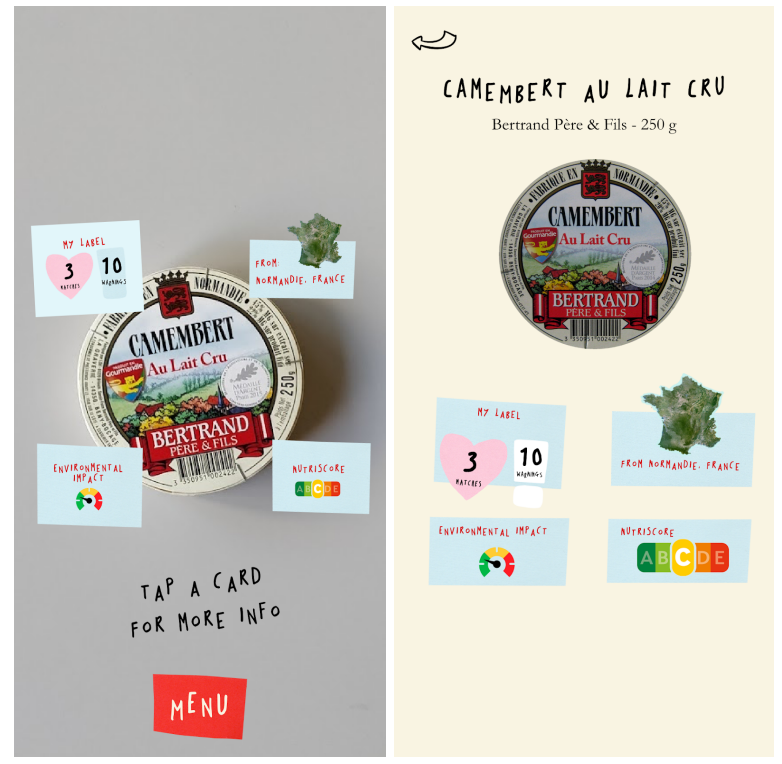

Figure 2: The AR version (left) and static version (right)

We created a functional prototype that worked with 5 different supermarket products. We created one version of the app that showed the information in AR, and for evaluation purposes, a second version of the app which showed the same information, with the same design, but using a static page that appears after a product is scanned.

\subsection{Study Method}

We set out to evaluate participants' recollection of information and to compare this between the AR and static versions of the application. In addition, we wanted to evaluate usability (performance, satisfaction, affective state, aesthetics) and compare this in both versions. In order to develop design guidelines, we also wanted to gather qualitative feedback on positive and negative aspects of the application. Finally, we wanted to control for how long participants use the app, how much money participants normally spend on shopping, how often participants buy organic products and participant awareness of ecological issues. 


\subsubsection{Participants}

84 participants were recruited, 64 of which were students from the University of Fribourg and 20 from the general population. There were no preconditions for recruitment. The age of the participants ranged from 17 to 54 , with the mean age being 23.23 years $(\mathrm{SD}=$ 6). $69 \%$ of the participants were female.

\subsubsection{Procedure}

Participants were asked to complete several pre-test questionnaires using a provided laptop before using the application. Participants were then told to imagine that they had just come home with a bag of shopping and wanted to look at five food products (with diverse packaging) with the application on a OnePlus 5T smartphone. They were told they could take as much time as they needed to do this. Participants were randomly assigned to the experimental groups, with 43 using the AR version and 41 using the static version. The experimentation took place within a university study room decorated to look like a home. Their behaviour was remotely observed using a (non-recorded) video transmission of the testing room from a YI 4K+ camera and a recorded transmission of their actions on the phone screen using TeamViewer software. Once they had finished, they were then asked to complete a further questionnaire and a quiz using a provided laptop.

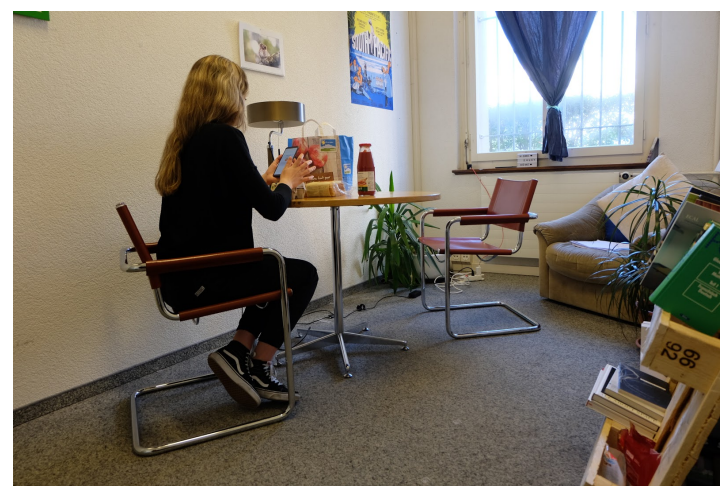

Figure 3: The testing scenario

\subsubsection{Assessment tools}

To measure affective state, we used the Animated SelfAssessment Manikin (AniSAM). This is an animated pictorial nonverbal evaluation technique directly measures the valence and arousal associated with the emotional response of a person exposed to a wide variety of stimuli [25]. To measure aesthetics, we used the Visual Aesthetics of Websites Inventory, short (VisAWI-s) by Moshagen \& Thielsch [19]. To measure usability, we used the System Usability Scale (SUS) [2], a scale commonly used in UX practice to measure subjective satisfaction with an interactive system. In order to assess how much the participants learned by using the application, we asked them questions about the five test products before and after using the application. They were asked to rank each product on a scale of 1 to 5 for nutritional value and a scale of 1 to 10 for environmental impact.

\subsection{Results}

When comparing the two versions of the application (AR vs static) there is a main effect with regard to the knowledge test after using the application $(\mathrm{F}(1,78)=4.8, \mathrm{p}<.05$, eta2 $=.06)$ with AR users learning more from the application than static users (when controlling for knowledge score before using the application and their awareness of ecological issues) (c.f. Figure
4). These covariates are evaluated with the following values; environmental awareness $=3.51$ and knowledge before using the application $=0.2595$. We controlled for knowledge score because, for unknown reasons, the group using the static version of the app had a significantly higher knowledge of products before looking at the application. We controlled for environmental awareness as we believed that existing knowledge and interest of participants could impact their scores.

The results also show that those using the AR application spent longer looking at products $(\mathrm{M}=412$ seconds, $\mathrm{SD}=195)$ compared to those using the static version $(\mathrm{M}=359$ seconds, $\mathrm{SD}$ $=114 ; \mathrm{F}=4.33, \mathrm{p}<.05, \eta 2 \mathrm{P}=.054)$.

The analysis of the subjective data (c.f. table 1) revealed no effects of the application version (AR vs static) with regards to usability (SUS) all Fs $<1$ and aesthetics (VisAWI-s) (all Fs $<1$ ). However, both versions were evaluated highly with regard to their usability with a combined mean SUS score of 86.4 (SD = 10.8), representing an evaluation ranging within the best $4 \%$ according to norming data [23]. In addition, with regard to visual aesthetics, both versions of the application scored highly with combined mean VisAWI-s value of $5.9(\mathrm{SD}=1.0)$, where a value of 4.5 is considered a positive benchmark [19].

For the statistical analysis of measures of affective states (valence and arousal), we controlled for the baseline measure of the two variables. Results indicate that there were no differences in affective reaction between the two versions of the app (both Fs $<1)$. Participants ranked the usefulness of being able to personalise their food label with a mean of $5.9(\mathrm{SD}=2.9)$ on a scale of 1 to 10 .

An analysis of the qualitative comments from users showed that they valued ease of use, scanning speed, the information overview, provenance information, and environmental information and that improvements could be made by having more information, improving scanning stability, and giving more explanation of environmental information.

\begin{tabular}{|c|l|l|l|l|}
\hline & Condition & Mean & SD & N \\
\hline \multirow{4}{*}{ SUS } & AR & 85.868 & 11.3040 & 38 \\
\cline { 2 - 5 } & Static & 86.865 & 10.4348 & 37 \\
\cline { 2 - 5 } & Total & 86.360 & 10.8218 & 75 \\
\hline VisAWI-s & AR & 5.8855 & .95195 & 38 \\
\cline { 2 - 5 } & Static & 5.9973 & 1.06340 & 37 \\
\cline { 2 - 5 } & Total & 5.9407 & 1.00319 & 75 \\
\hline Learning & AR & 0.5395 & 0.16495 & 43 \\
\cline { 2 - 5 } & Static & 0.4707 & 0.23690 & 41 \\
\cline { 2 - 5 } & Total & 0.5060 & 0.20497 & 84 \\
\hline \multirow{2}{*}{$\begin{array}{l}\text { Time spent } \\
\text { using the app } \\
\text { in seconds }\end{array}$} & AR & 412.48 & 195.03 & 41 \\
\cline { 2 - 5 } & Static & 359.05 & 113.83 & 39 \\
\cline { 2 - 5 } & Total & 386.43 & 161.91 & 80 \\
\hline
\end{tabular}

Table 1. Means, standard deviations (SD) and sample sizes $(N)$ for the different variables for the $A R$ and Static versions of the Food Talks application 


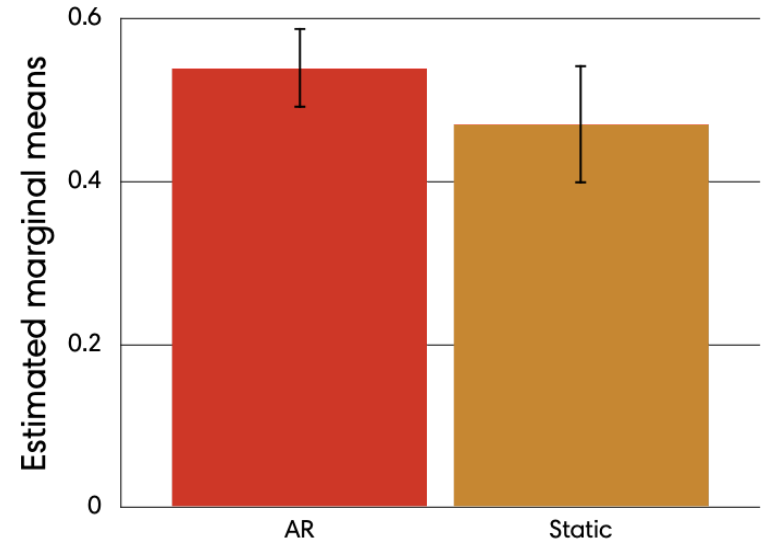

\section{Condition}

Figure 4: Estimated marginal means of knowledge after using the AR or Static application. Error bars $95 \% \mathrm{Cl}$.

\section{Discussion}

\subsection{The value of Augmented Reality}

We showed that participants using our AR smartphone application learned more than those using an equivalent static version of the application. This shows that they read and understood the content, despite the potential for them to be distracted by the "wow effect", or technical novelty of the AR.

We propose the following hypotheses to support this main result. The first is that having information directly next to the food product in space creates a stronger link between the two, thus aiding people's recollection, although the data obtained in this study do not allow to confirm this assumption. The second hypothesis is based on our finding that participants spent longer using the AR application. The reasons for spending longer in AR could be due to an increased curiosity and engagement with the interface that has more intriguing properties than the Static version.

\subsection{Aesthetics, usability and affective state}

The results of the SUS and VisAWI-s questionnaires reveal that there was no significant difference between showing the information in AR versus on a static page with regards to usability and aesthetics. This shows that despite the technical issues reported for AR relating to scanning reliability, in the end, our AR design was considered to have the same usability and aesthetics as the static version. In addition, the fact that we found no difference between the AR and Static versions of the application regarding affective state could indicate that our design allowed participants to not be disturbed by the novelty of the technology.

\subsection{Information Design}

The positive results for usability and aesthetics as well as the qualitative feedback on the overview layout of the information, indicates the effectiveness of the design to present nutritional and environmental information together in AR. The final design was based on learnings from regular testing with users in Phase 1 and also employed principles from the literature. Indeed, this indicates that the design principles learned from the public Kiosk in Phase 1 were able to be transferred to the mobile AR scenario at home.

Although participants were pleased to have environmental information some still had problems interpreting the parameters and units of measure. As there were limited precedents to how to best show this information there is still room for improvement.

Design learnings from this project are detailed below.

\subsubsection{Design learnings}

- Using a modular structure allows information to be traded in and out, and can therefore work with large database without the need for redesign.

- Using an overview of several information parameters gives a summary and equal hierarchy to all information modules.

- Using minimal numbers as well as colours, symbols and icons makes information identifiable.

- Using a collage, or non-photorealistic style allows "super normal", familiar and coherent integration of augmented digital content with the real-world scene.

- The addition of graphic links between the digital interface and augmented content further strengthens the coherence of the system.

- Using sound effects where appropriate also contributes to more coherent AR content.

- An adaptive interface colour scheme that matches food product packaging colours strengthens the link between the digital content and the target object.

- Putting a "billboard" background behind text aids legibility in a range of environments.
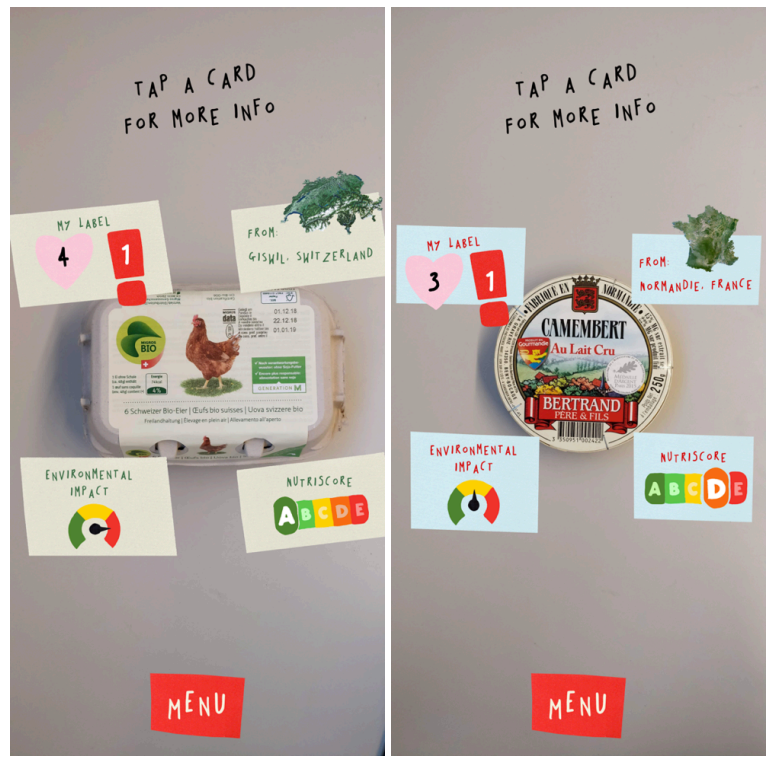

Figure 5: The mobile AR design showing the adaptive interface colours, billboard background, icons and collage style

\subsection{Technological Feasibility}

Our positive results for usability, aesthetics and learning show the potential of AR to be used in the context of food labelling. However, our study was conducted on a small scale with a controlled number of food products. Some of the qualitative issues reported with the reliability of AR scanning highlight the fact that using AR on a large scale with many supermarket products might not be feasible with the current state of the technology. 


\subsection{Study design}

Unlike the majority of AR usability studies which are withinsubjects and have a mean value of 18 participants [4], our study followed a between-subjects design based on a large sample size of 84 participants.

This initial UX evaluation of our prototype was important to test the scope of AR in the context of food labelling. It also adds UX evaluation knowledge to the field of AR. However, in future research, it would be useful to conduct a longitudinal study [26] addressing other aspects such as the adoption of AR and differences between the two design variations. This would give insights into which variation was preferred over the long term.

\section{Conclusion}

The results from this project contribute several learnings. Firstly, our two-phase process enabled us to create two prototypes adapted to two environments and type of users, but also a singular and effective way to present different parameters of information together using AR. Secondly, as our participants read and remembered the content they saw using $\mathrm{AR}$ and were not distracted by the technical complexity, we were able to show that AR can be a credible medium to communicate data. Thus, AR has the potential to be used in the food sector beyond short-term advertising campaigns that focus only on the spectacular effect of the technology. Thirdly, our results confirm that there is a public interest to have more information about food products than currently exists. In particular, we showed that the new additions of environmental, provenance and personalisation have value. Finally, we share our learnings with other designers. Future work should undertake longitudinal studies and aim to maximise AR scanning reliability.

\section{REferences}

[1] Nadine Bayle. 2018. Les marques à la merci des applis de notation. Retrieved March 27, 2019 from https://www.lemonde.fr/long-

format/article/2018/11/04/consommation-les-marques-a-lamerci-des-applis_5378697_5345421.html

[2] John Brooke. 1996. SUS-A quick and dirty usability scale. Usability Eval. Ind. 189, 194 (1996), 4-7.

[3] Sarah Campos, Juliana Doxey, and David Hammond. 2011. Nutrition labels on pre-packaged foods: a systematic review. Public Health Nutr. 14, 8 (August 2011), 14961506. DOI:https://doi.org/10.1017/S1368980010003290

[4] Rachael L Dettmann and Carolyn Dimitri. 2009. Who's buying organic vegetables? Demographic characteristics of US consumers. J. Food Prod. Mark. 16, 1 (2009), 79-91.

[5] Arindam Dey, Mark Billinghurst, Robert W. Lindeman, and J. Edward II Swan. 2018. A Systematic Review of 10 Years of Augmented Reality Usability Studies: 2005 to 2014. Front. Robot. AI 5, (2018). DOI:https://doi.org/10.3389/frobt.2018.00037

[6] Manon Egnell, Pauline Ducrot, Mathilde Touvier, Benjamin Allès, Serge Hercberg, Emmanuelle KesseGuyot, and Chantal Julia. 2018. Objective understanding of Nutri-Score Front-Of-Package nutrition label according to individual characteristics of subjects: Comparisons with other format labels. PLOS ONE 13, 8 (August 2018), e0202095.

DOI:https://doi.org/10.1371/journal.pone.0202095

[7] Stephen R Ellis, Francois Breant, B Manges, Richard Jacoby, and Bernard D Adelstein. 1997. Factors influencing operator interaction with virtual objects viewed via head-mounted see-through displays: viewing conditions and rendering latency. 138-145.

[8] J. Fischer, D. Bartz, and W. Straber. 2005. Stylized augmented reality for improved immersion. In IEEE Proceedings. VR 2005. Virtual Reality, 2005., 195-202. DOI:https://doi.org/10.1109/VR.2005.1492774

[9] Naoto Fukasawa and Jasper Morrison. 2007. Super normal: Sensations of the ordinary. Lars Müller.

[10] Chris Furmanski, Ronald Azuma, and Mike Daily. 2002. Augmented-reality visualizations guided by cognition: Perceptual heuristics for combining visible and obscured information. 215-320.

[11] J. L. Gabbard, J. E. Swan, and D. Hix. 2006. The Effects of Text Drawing Styles, Background Textures, and Natural Lighting on Text Legibility in Outdoor Augmented Reality. Presence 15, 1 (February 2006), 16-32. DOI:https://doi.org/10.1162/pres.2006.15.1.16

[12] Zaina Gadema and David Oglethorpe. 2011. The use and usefulness of carbon labelling food: A policy perspective from a survey of UK supermarket shoppers. Food Policy 36, 6 (2011), 815-822.

[13] Klaus G Grunert, Josephine $M$ Wills, and Laura Fernández-Celemín. 2010. Nutrition knowledge, and use and understanding of nutrition information on food labels among consumers in the UK. Appetite 55, 2 (2010), 177189.

[14] Michael Haller. 2004. Photorealism or/and nonphotorealism in augmented reality. 189-196.

[15] N. Henchoz, V. Lepetit, P. Fua, and J. Miles. 2011. Turning Augmented Reality into a media: Design exploration to build a dedicated visual language. In 2011 IEEE International Symposium on Mixed and Augmented Reality - Arts, Media, and Humanities, 83-89. DOI:https://doi.org/10.1109/ISMAR-AMH.2011.6093661

[16] Ernst Kruijff, J Edward Swan, and Steven Feiner. 2010. Perceptual issues in augmented reality revisited. In 9th IEEE International Symposium on Mixed and Augmented Reality 2010: Science and Technology, ISMAR 2010 Proceedings, DOI:https://doi.org/10.1109/ISMAR.2010.5643530

[17] Allison M. Leach, Kyle A. Emery, Jessica Gephart, Kyle F. Davis, Jan Willem Erisman, Adrian Leip, Michael L. Pace, Paolo D'Odorico, Joel Carr, Laura Cattell Noll, Elizabeth Castner, and James N. Galloway. 2016. Environmental impact food labels combining carbon, nitrogen, and water footprints. Food Policy 61, C (2016), 213-223.

[18] Mark A Livingston, J Edward Swan, Joseph L Gabbard, Tobias H Hollerer, Deborah Hix, Simon J Julier, Yohan Baillot, and Dennis Brown. 2003. Resolving multiple occluded layers in augmented reality. 56-65.

[19] Morten Moshagen and Meinald Thielsch. 2013. A short version of the visual aesthetics of websites inventory. Behav. Inf. Technol. 32, 12 (2013), 1305-1311.

[20] Tobias Müller. 2019. Challenges in representing information with augmented reality to support manual procedural tasks. ElectrEng 2019 Vol 3 Pages 71-97 (January DOI:https://doi.org/10.3934/ElectrEng.2019.1.71

[21] Nielsen. 2016. What's in Our Food and on Our Mind: Ingredient and Dining-Out Trends Around the World. Retrieved March 28, 2019 from https://www.nielsen.com/content/dam/nielsenglobal/eu/doc s/pdf/Global\%20Ingredient $\% 20$ and $\% 20$ Out-ofHome\%20Dining\%20Trends\%20Report.pdf 
[22] Kajsa Palm. 2018. Design and use of 3D typography for indoor Augmented Reality mobile applications. Retrieved June 26, 2018 from http://urn.kb.se/resolve?urn=urn:nbn:se:umu:diva-148619

[23] Megan E. Rollo, Tamara Bucher, Shamus P. Smith, and Clare E. Collins. 2017. ServAR: An augmented reality tool to guide the serving of food. Int. J. Behav. Nutr. Phys. Act. 14, 1 (May 2017), 65. DOI:https://doi.org/10.1186/s12966017-0516-9

[24] Jessica Savage and Christina B Johns. 2015. Food allergy: epidemiology and natural history. Immunol. Allergy Clin. 35, 1 (2015), 45-59.

[25] Andreas Sonderegger, Klaus Heyden, Alain Chavaillaz, and Juergen Sauer. 2016. AniSAM \& AniAvatar: Animated Visualizations of Affective States. In Proceedings of the 2016 CHI Conference on Human Factors in Computing Systems (CHI '16), 4828-4837. DOI:https://doi.org/10.1145/2858036.2858365

[26] Andreas Sonderegger, Gerold Zbinden, Andreas Uebelbacher, and Juergen Sauer. 2012. The influence of product aesthetics and usability over the course of time: a longitudinal field experiment. Ergonomics 55, 7 (July 2012), $713-730$. DOI:https://doi.org/10.1080/00140139.2012.672658

[27] Natsuki Sugano, Hirokazu Kato, and Keihachiro Tachibana. 2003. The effects of shadow representation of virtual objects in augmented reality. 76-83.

[28] Ryosuke Tanno, Takumi Ege, and Keiji Yanai. 2018. AR DeepCalorieCam: An iOS App for Food Calorie Estimation with Augmented Reality. In MultiMedia Modeling (Lecture Notes in Computer Science), 352-356.

[29] Daniel Wagner, Mark Billinghurst, and Dieter Schmalstieg. 2006. How Real Should Virtual Characters Be? In Proceedings of the 2006 ACM SIGCHI International Conference on Advances in Computer Entertainment Technology (ACE '06). DOI:https://doi.org/10.1145/1178823.1178891

[30] Christian Wallraven and Heinrich Bülthoff Wolfgang Straßer. 2006. Measuring the discernability of virtual objects in conventional and stylized augmented reality. 53 .

[31] 2018. Why people in rich countries are eating more vegan food. The Economist. Retrieved March 28, 2019 from https://www .economist.com/briefing/2018/10/13/whypeople-in-rich-countries-are-eating-more-vegan-food

[32] 18 Examples of AR Packaging. TrendHunter.com. Retrieved May 1, 2019 from https://www.trendhunter.com/slideshow/ar-packaging

[33] Yuka - Product scan - Apps on Google Play. Retrieved April 26, 2019 from https://play.google.com/store/apps/details?id=io.yuka.andr oid\&hl=en_AU

[34] CodeCheck: Food \& Cosmetics Scanner - Apps on Google Play. Retrieved April 26, 2019 from https://play.google.com/store/apps/details?id=ch.ethz.im.co decheck\&hl=en_AU

[35] Open Food Facts - Apps on Google Play. Retrieved April 26, 2019 from https://play.google.com/store/apps/details?id=org.openfood facts.scanner\&hl=en_AU

[36] ecoinvent. Retrieved January 17, 2018 from http://www.ecoinvent.org/

[37] Quantis I WFLDB - World Food Life Cycle Assessment Database. Quantis. Retrieved January 17, 2018 from https:/quantis-intl.com/tools/databases/wfldb-food/ 